\title{
Investigation on Two-Stop-Line Signalized Roundabout: Capacity and Optimal Cycle Length
}

\author{
Ze-hao Jiang $\mathbb{D}^{1,}, 2$ Tao Wang $\mathbb{D}^{1},{ }^{3}$ Chao-yang Li, ${ }^{3}$ Fei Pan, ${ }^{1}$ and Xiao-guang Yang $\mathbb{D}^{1}$ \\ ${ }^{1}$ Key Laboratory of Road and Traffic Engineering, Ministry of Education, Tongji University, Shanghai 200092, China \\ ${ }^{2}$ Department of Civil and Earth Resources Engineering, Kyoto University, Kyoto 606-8501, China \\ ${ }^{3}$ School of Naval Architecture, Ocean \& Civil Engineering, Shanghai Jiao Tong University, Shanghai 200240, China
}

Correspondence should be addressed to Ze-hao Jiang; 528jzh@tongji.edu.cn and Tao Wang; wt127@sina.com

Received 9 February 2019; Revised 24 April 2019; Accepted 8 May 2019; Published 29 May 2019

Academic Editor: David F. Llorca

Copyright (C) 2019 Ze-hao Jiang et al. This is an open access article distributed under the Creative Commons Attribution License, which permits unrestricted use, distribution, and reproduction in any medium, provided the original work is properly cited.

\begin{abstract}
Two-stop-line signalized roundabouts (TSLSR) are widely utilized in China. To calculate the capacity and optimal cycle length accurately, a model considering internal space constraint of the roundabout is developed in this paper. Firstly, the operational principle of TSLSR is analyzed. TSLSR is modeled as being equivalent to a four-phase intersection with left-turn protection phases, and the left-turn lane is further simplified as the "short-lane model". Secondly, based on the above analysis, the capacity of TSLSR is modeled and additionally, the accuracy and sensitivity of the model are also analyzed. Finally, the optimal cycle length of TSLSR which maximizes the capacity is put forward. Results show that the accuracy of the capacity model is low without considering the internal space constraint of the roundabout. However, the model developed in this paper is shown to improve the accuracy (about $20 \%$ ) and control the relative error to be within $10 \%$. The parameter sensitivity analysis demonstrates that the cycle length and radius of the central island have a significant influence on the capacity of the left-turn lane and results in an optimal value of signal cycle length maximizing the capacity.
\end{abstract}

\section{Introduction}

The circular configuration of roundabouts requires vehicles to circulate in a counterclockwise direction (in the China and other right-hand traffic countries) and has been employed successfully to change conflict points from crossing conflicts to merging conflicts. They also help reduce traffic speeds as a result of their deflection angle on entry and circular configuration. The reduced vehicle speeds and motor vehicle conflicts are the reasons why roundabouts are considered a traffic safety enhancement method. According to Highway Safety Manual [1], the safety will be significantly improved if a signalized intersection/stop-controlled intersection is converted to a modern roundabout. For example, in urban areas, if a single-lane or two-lane signalized intersection is converted to a modern roundabout, the injury crash modification factor (CMF) is 0.40 , which means the expected number of injuries would decrease by $60 \%$ [1]. In addition, roundabouts always have good landscape effects and are often geographical symbols of the cities. Because of the above advantages, roundabouts have been widely used in China.

With the continuous development of economy and rapid growth of vehicle ownership in China, traffic congestion is getting increasingly worse in Chinese cities. However, many of China's roundabouts were built decades ago and are located in the city center areas. As a result, roundabouts are often congestion points of road networks.

Since the entering traffic must yield to the circulating traffic and then wait for a gap to enter the roundabout, it is uncommon to have roundabouts carrying more than 3,000 vehicles per hour [2]. To break through the capacity limit, there are two feasible options. The first approach is to convert a roundabout to a signalized intersection or an interchange. This option usually has high construction costs with serious impacts on the operation of the road network. The second choice is to only repaint the traffic marks and equip traffic signals at the roundabout to enhance the capacity. This 


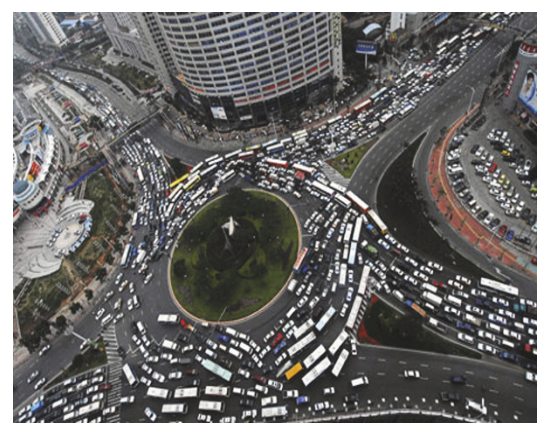

Before

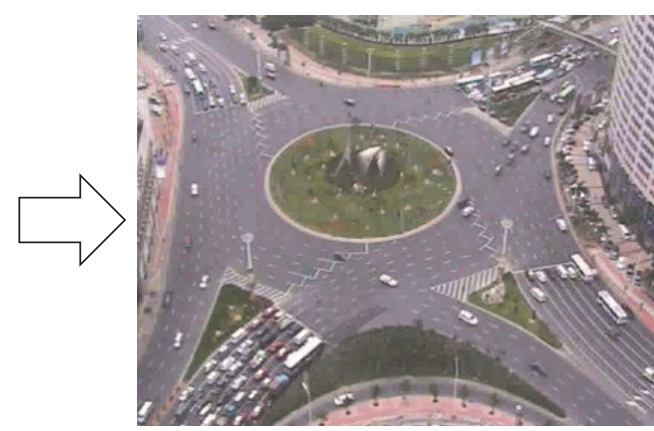

After

FIGURE 1: A before-and-after scenario by using the TSLSR.

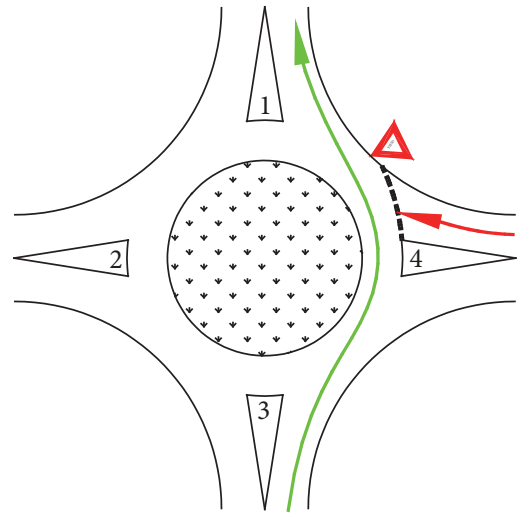

Modern roundabout

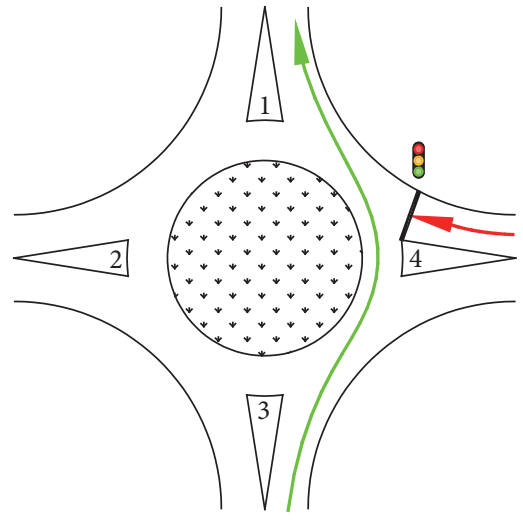

ASCR

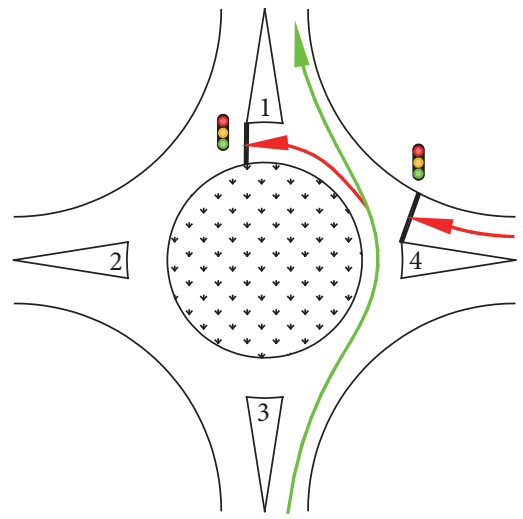

TSLR

FIgURE 2: The schematic diagram of modern roundabout, ASCR, and TSLR.

method has been proven by some engineering projects led by us as a cost-effective way to handle high traffic demands. Figure 1 shows a case from Xiamen City, China, in which the looking up and traffic congestion during the peak hour at the roundabout have been significantly alleviated.

This approach is called "two-stop-line signalized roundabout (TSLSR)" in this article. Its significant advantages are low transformation costs, short construction time, and limited impact on urban road networks. In addition, the signal control scheme can be flexibly adjusted according to changes in traffic volume.

In view of the importance of TSLSR in certain engineering applications, this research aims to investigate an engineering-oriented approach to calculate the capacity and optimal cycle length of TSLSR. The remainder of this paper is organized as follows. We first provide a literature review in Section 2. Section 3 demonstrates the basic operating principle of TSLSR. An engineering-oriented capacity calculation model of TSLSR is discussed in Section 4, followed by numerical sensitivity and precision analysis in Section 5 . The calculation method of optimal cycle length maximizing the capacity is then presented in Section 6. Finally, Section 7 concludes this study.

\section{Background Literature}

In this section we provide a description of modern roundabouts, and then conduct a literature review of approachsignal-control roundabout (ASCR) and two-stop-line signalized roundabout (TSLSR).

A modern roundabout [3] is a form of intersection in which the entering traffic must yield to the circulating traffic. In 1966, the UK adopted a rule, known as the "priorityto-the-circle rule" [4], that required entering traffic to give way to circulating traffic at all circular intersections. This rule prevented roundabouts from locking up by not allowing vehicles to enter the intersection until there were sufficient gaps in circulating traffic. In this way, the entrance lanes of modern roundabouts are regarded as the minor road and the circulatory lanes as the major road. The roundabout can thus be considered as a typical stop-controlled intersection [5]. Hence, the entrance capacity [6], delay [7], and queue length [8] can be calculated using the gap acceptance theory. A number of articles $[6,9,10]$ provide detailed literature reviews on the capacity models of modern roundabouts.

ASCRs are circular intersections where traffic signals are equipped to control vehicles stopping before the approach stop line, shown as dark bold line alongside the traffic light in Figure 2, ASCR. This idea was proposed in 1970s 


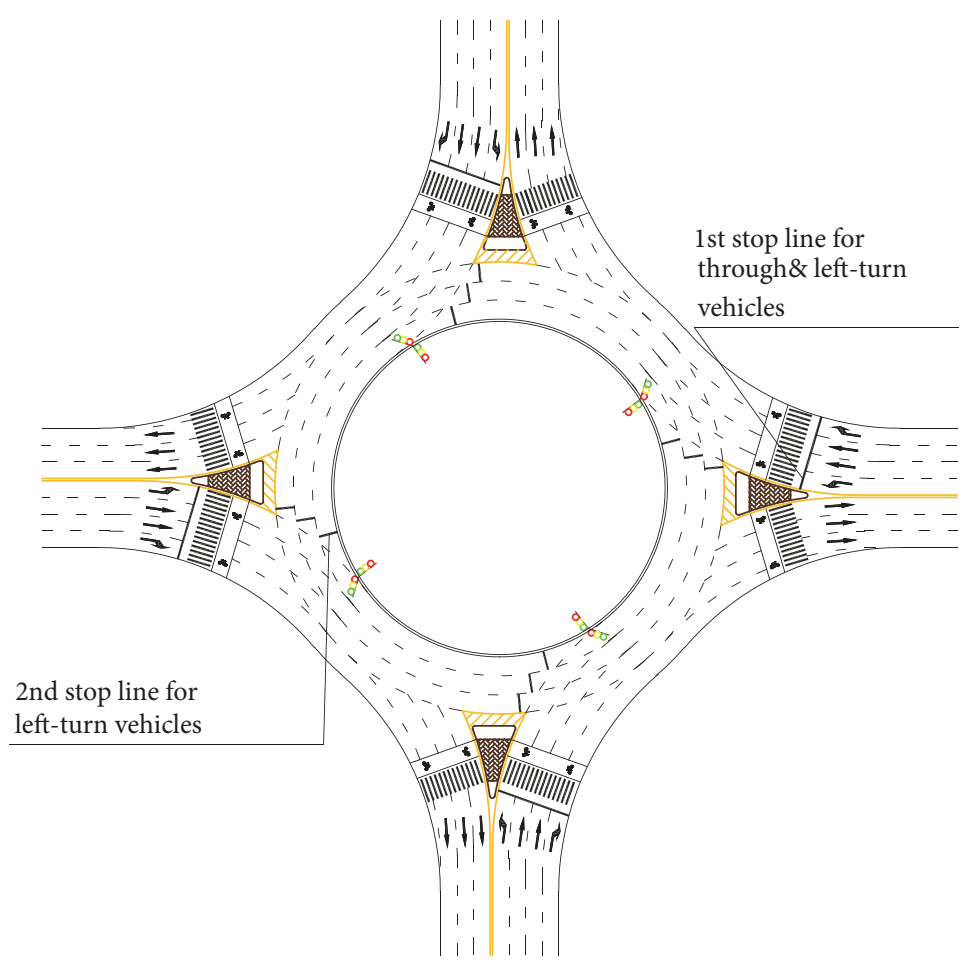

FIGURE 3: The typical geometric layout of a TSLSR.

[11]. As a result, ASCRs have distinctly different operational characteristics from modern roundabouts. Since then, a series of studies demonstrated that adding signals at the approaches of roundabouts can enhance the capacity [12], eliminate the locking up, and reduce $\mathrm{CO}_{2}$ emissions $[13,14]$. In order to calculate the capacity of ASCR, HCM (Highway Capacity Manual) method is usually employed in which the basic formulation is the saturation flow rate multiplied by the effective green time [12]. However, traffic delays and formation of long queues at the approaches will still occur because the spaces of circulatory lane inside the ASCRs are not fully utilized.

To make full use of the internal space of ASCR, TSLSR was invented and widely used in China [15], but has not been promoted abroad. TSLSR has been verified by engineering projects and proved as a cost-efficient solution to enhance the roundabout capacity. The key characteristics and operating principle of TSLSR will be introduced in Section 3. Ma et al. [16] did a summative study on the integrated optimization of lane making and timing for TSLSR and proved TSLSR outperforms the signalized intersections and modern roundabouts in most cases. However, in this research, to represent the roundabout phase diagram, a precedence graph was developed, which is rather complicated and difficult for engineering applications.

\section{Operating Principle of TSLSR}

In this section the operational logic of TSLSR is first introduced. Then TSLSR is simplified into a classic four-phase signalized intersection, in which the left-turn lane can be further simplified as the "short-lane model". The discussion in this section is limited to symmetric four-arm roundabouts, but it could be easily extended to TSLSR of other forms, symmetric or not symmetric, with more or less than four arms.

3.1. Operational Logic. The typical geometric layout of a TSLSR with symmetric four arms is shown in Figure 3. TSLSR is featured with two stop lines and two sets of traffic signals.

(i) Two stop lines: the first stop lines are painted at the approaches (see 1st stop line for through and left-turn vehicles in Figure 3) and the second stop lines are at the circulatory lanes (see 2nd stop line for left-turn vehicles in Figure 3).

(ii) Two sets of traffic signals: the signals are named as the main signal and presignal, respectively. The one in front of the 1st stop line is the main signal and that in front of the 2 nd stop line is presignal.

Figure 4 illustrates the typical signal phase diagram of a TSLSR. The basic operating logic is as follows:

(i) At the approaches of TSLSR, left-turn vehicles and through vehicles utilize different lanes and are controlled by different signal lights.

(ii) Left-turn vehicles should stop twice. The first time is at the 1st stop line for through and left-turn vehicles and the second time is at the 2 nd stop line for left-turn vehicles (see P-2 and P-3 in Figure 4). 

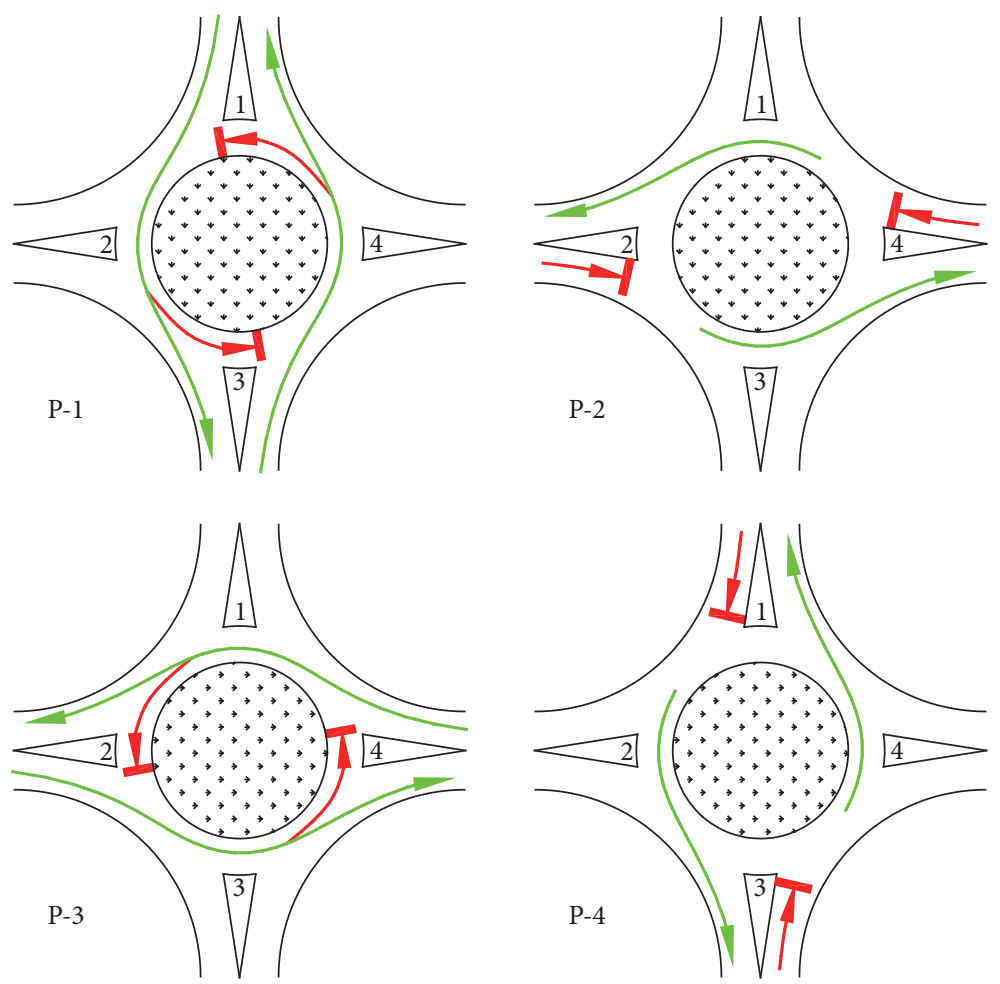

FIgURE 4: The typical signal phase diagram of a TSLSR.

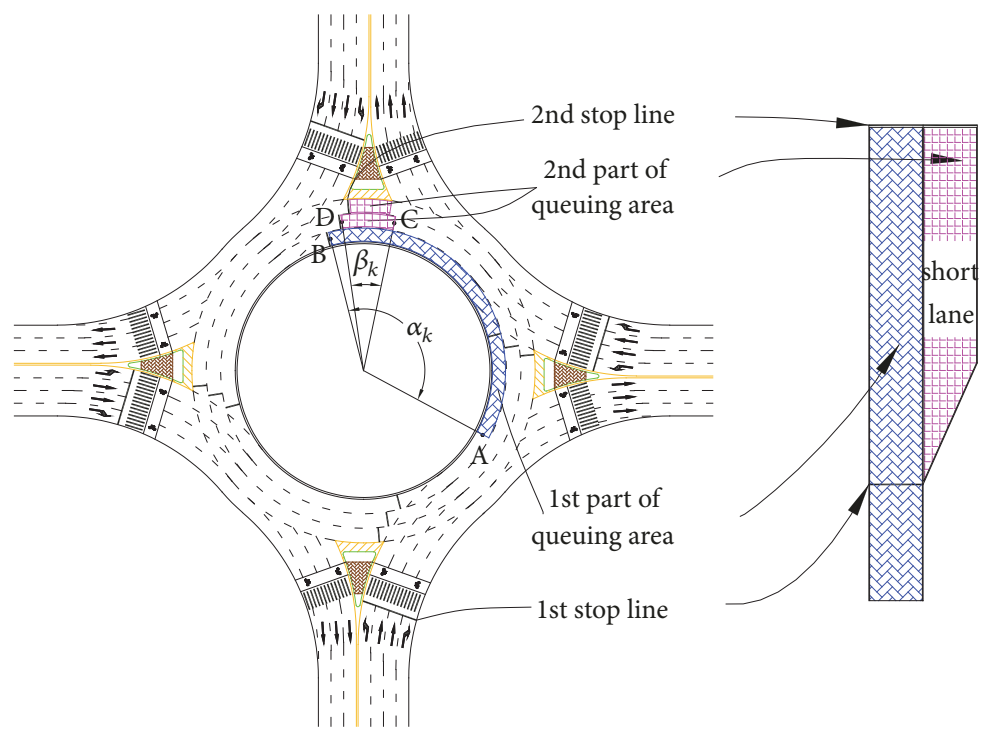

FIGURE 5: Short-lane analysis of right-turn lane.

3.2. Equivalent Simplification as a Short-Lane Model. The above analysis indicates that, from the viewpoint of traffic signal control, the operational logic of TSLSR can be essentially regarded as equivalent to a classic four-phase intersection with left-turn protection phases. Moreover, considering the interval space constraint of circulatory lanes, as shown in Figure 5, the TSLSR can be further simplified as "a classic four-phase intersection with right-turn short lanes”.
The current research on the capacity of the short lane is relatively mature. In a study of Tian et al. [17], they found that the capacity of a signalized intersection with a short rightturn lane is significantly related to the length of the short lane, the proportion of through and right-turn vehicles, and the cycle length. Ma et al. [18] built a probiotic model to calculate the capacity of signalized intersections with short lane, in which three block scenarios including no blockage, probable blockage, and after blockage were taken into consideration. 
TABLE 1: Variable notations in this research.

\begin{tabular}{|c|c|c|}
\hline Variable notations & Description & Unit \\
\hline$C_{t}$ & Capacity of one through lane at the 1st stop line & $\mathrm{pcu} / \mathrm{h}$ \\
\hline$C_{l 1}$ & Capacity of one left-turn lane at the 1st stop line & $\mathrm{pcu} / \mathrm{h}$ \\
\hline$C_{l 2}$ & Capacity of one left-turn lane at the 2 nd stop line & $\mathrm{pcu} / \mathrm{h}$ \\
\hline$C_{l}$ & Overall capacity of one left-turn lane & $\mathrm{pcu} / \mathrm{h}$ \\
\hline$C$ & Overall capacity of one direction approach & $\mathrm{pcu} / \mathrm{h}$ \\
\hline$s_{t 1}$ & Saturation flow of one through lane at the 1st stop line & $\mathrm{pcu} / \mathrm{h}$ \\
\hline$s_{l 1}$ & Saturation flow of one left-turn lane at the 1st stop line & $\mathrm{pcu} / \mathrm{h}$ \\
\hline$g_{t 1}$ & Effective green time for through traffic flow & s \\
\hline$g_{l 1}$ & Effective green time for left-turn traffic flow at 1st stop line & $\mathrm{s}$ \\
\hline$T$ & Cycle length of the traffic signal & $\mathrm{s}$ \\
\hline$T_{\text {opt }}$ & Optimal cycle length of the traffic signal & s \\
\hline$C_{l 2}^{\prime}$ & Vehicle-storage capacity of $1^{\text {st }}$ and $2^{\text {nd }}$ part of queueing area & pcu \\
\hline$m$ & Number of circulatory lanes assigned to left-turn traffic flow during phase P-2 & \\
\hline$n$ & Total number of circulatory lanes excluding right-turn lane & \\
\hline$\alpha_{k}$ & Radian of the 1st part of queuing area & $\mathrm{rad}$ \\
\hline$\beta_{k}$ & Radian of the 2nd part of queuing area & $\mathrm{rad}$ \\
\hline$R$ & Radius of the central island & $\mathrm{m}$ \\
\hline$R_{k}$ & Radius of circulatory lane $k$ & $\mathrm{~m}$ \\
\hline$l_{v}$ & Average parking space for a vehicle, normally is 6 & $\mathrm{~m}$ \\
\hline$q_{i}$ & Traffic volume of lane $i$ & $\mathrm{pcu} / \mathrm{h}$ \\
\hline$d_{i}$ & Average traffic delay of lane $i$ & $\mathrm{~s}$ \\
\hline
\end{tabular}

\section{Capacity Model}

In this section, we aim to put forward a model to calculate the capacity of TSLSR; particularly the internal space is considered. Left-turn lanes and through lanes are the two main concerns in the following analysis, as the right-turn vehicles are normally not controlled by signals and free to pass.

To facilitate the presentation, key variable notations used hereafter in the paper are summarized in Table 1.

4.1. Capacity at the 1st Stop Line. The through traffic flow is only controlled by the signal lights equipped at the 1st stop line. Hence the roundabout after passing the 1st stop line without ever stopping before the 2 nd stop line exits. As a result, the capacity of through traffic flow can be calculated using HCM method [19], as shown in (1):

$$
C_{t}=s_{t 1} \frac{g_{t 1}}{T}
$$

On the other hand, left-turn vehicles need to pass through both the 1st and 2nd stop lines before leaving the roundabout. As a result, the overall capacity of left-turn traffic flow is the minimum capacity of the two stop lines, as shown in (2):

$$
C_{l}=\min \left(C_{l 1}, C_{l 2}\right)
$$

Similar to the through traffic flow capacity at the 1st stop line, the capacity of the left-turn traffic flow at 1st stop line can be given by (3):

$$
C_{l 1}=s_{l 1} \frac{g_{l 1}}{T}
$$

4.2. Capacity at the 2nd Stop Line. When the left-turn signal at the 2nd stop line turns green, the signal upstream of it, which is the left-turn signal at the 1st stop line, will turn red as shown in phases P-2 and P-4 in Figure 4. In other words, when the left-turn vehicles are passing the 2 nd stop line, the corresponding 1st stop line will be shut down from releasing traffic flow. Therefore, in any given signal cycle, the maximum number of vehicles passing through the 2nd stop line equals to the vehicle-storage capacity of 1st and 2nd part of queuing areas. These two queuing areas are mainly affected by the number of circulatory lanes assigned to left-turn traffic flow. According to the basic geometry knowledge, the vehiclestorage capacity of 1st and 2nd part of queuing areas (shown in Figure 4) can be easily calculated by (4):

$$
C_{l 2}^{\prime}=\sum_{k=1}^{m} \frac{\alpha_{k} \cdot R_{k}}{l_{v}}+\sum_{k=m+1}^{n} \frac{\beta_{k} \cdot R_{k}}{l_{v}}
$$

Then, the capacity of left-turn traffic flow at the 2 nd stop line can be calculated using (5):

$$
C_{l 2}=\frac{3600}{T} C_{l 2}^{\prime}=\frac{3600}{T}\left(\sum_{k=1}^{m} \frac{\alpha_{k} \cdot R_{k}}{l_{v}}+\sum_{k=m+1}^{n} \frac{\beta_{k} \cdot R_{k}}{l_{v}}\right)
$$

It should be noted here that, in China's municipal design code (CJJ-37, 2012), the length of a car is usually considered to be $6 \mathrm{~m}$. In fact, this length is larger than the actual value, but we consider that the vehicle needs to maintain a safe distance from the preceding vehicle when parking. Therefore, this value is still taken as $6 \mathrm{~m}$. 
TABLE 2: The accuracy test results.

\begin{tabular}{|c|c|c|c|c|c|c|}
\hline \multirow[t]{2}{*}{$\begin{array}{l}\text { Cycle } \\
\text { length }\end{array}$} & \multirow[t]{2}{*}{$\begin{array}{c}\text { VISSIM } \\
\text { simulation } \\
\text { (benchmark) }\end{array}$} & \multicolumn{2}{|c|}{$\begin{array}{l}\text { Model without } \\
\text { consideration of } \\
\text { interval space } \\
\text { constraint }\end{array}$} & \multicolumn{2}{|c|}{$\begin{array}{l}\text { model considering } \\
\text { the interval space } \\
\text { constraint } \\
\text { (proposed in this } \\
\text { paper) }\end{array}$} & \multirow[t]{2}{*}{$\begin{array}{c}\text { Improvement of model accuracy } \\
\text { after considering internal space } \\
\text { constraints }\end{array}$} \\
\hline & & Result & Relative error & Result & Relative error & \\
\hline 60 & 297 & 310 & $4.38 \%$ & 311 & $4.71 \%$ & $-0.34 \%$ \\
\hline 70 & 397 & 321 & $-19.13 \%$ & 378 & $-4.79 \%$ & $14.34 \%$ \\
\hline 80 & 438 & 329 & $-24.80 \%$ & 428 & $-2.28 \%$ & $22.52 \%$ \\
\hline 90 & 409 & 336 & $-17.89 \%$ & 466 & $13.94 \%$ & $3.95 \%$ \\
\hline 100 & 475 & 341 & $-28.21 \%$ & 498 & $4.84 \%$ & $23.37 \%$ \\
\hline 110 & 560 & 345 & $-38.35 \%$ & 522 & $-6.79 \%$ & $31.57 \%$ \\
\hline 120 & 517 & 349 & $-32.54 \%$ & 541 & $4.64 \%$ & $27.90 \%$ \\
\hline 130 & 525 & 352 & $-33.00 \%$ & 499 & $-4.95 \%$ & $28.05 \%$ \\
\hline 140 & 456 & 354 & $-22.31 \%$ & 463 & $1.54 \%$ & $20.77 \%$ \\
\hline 150 & 386 & 357 & $-7.64 \%$ & 431 & $11.66 \%$ & $-4.02 \%$ \\
\hline 160 & 437 & 358 & $-17.98 \%$ & 408 & $-6.64 \%$ & $11.34 \%$ \\
\hline
\end{tabular}

4.3. Overall Capacity of One Direction Approach. The overall capacity of the approach lanes in one direction includes the through lanes and left-turn lanes. This can be calculated by (6):

$$
\begin{aligned}
C= & C_{t}+C_{l}=s_{t 1} \frac{g_{t 1}}{T}+\min \left\{s_{l 1}\right. \\
& \left.\cdot \frac{g_{l 1}}{T},\left[\frac{3600}{T}\left(\sum_{k=1}^{m} \frac{\alpha_{k} \cdot R_{k}}{l_{v}}+\sum_{k=m+1}^{n} \frac{\beta_{k} \cdot R_{k}}{l_{v}}\right)\right]\right\}
\end{aligned}
$$

\section{Numerical Analysis}

5.1. Model Accuracy Test. In this section, the numerical model accuracy test and the sensitive analysis will be conducted.

Model accuracy verification is to investigate the degree of the model accuracy improvement when the internal space constraint of the TSLSR is taken into consideration. The model proposed in this paper will be compared with a model without the consideration of the internal space constraint. A model without the consideration of internal space constraint considers the capacity only at the $1^{\text {st }}$ stop line which equals to the saturation flow rate multiplied by the effective green time.

At the same time, since it is difficult to obtain the capacity value of TSLSR under different geometric layout and signal timing parameters, this paper employs the VISSIM simulation as the benchmark. The TSLSR was modeled by VISSIM [20] by using Figure 3 as the base image. The image was imported and scaled, and the links and connectors representing the roadway were then drawn. The roundabout priority rules were set by placing the conflict markers after which calibration was done by adjusting the priority rule parameters and minimum headways. The minimum headway of through lanes is $2.182 \mathrm{~s}$ which is equivalent to a saturation flow of $1650 \mathrm{pcu} / \mathrm{h}$. Similarly, the minimum headway of leftturn and right-turn lanes is $2.322 \mathrm{~s}$ which is equivalent to a saturation flow of $1550 \mathrm{pcu} / \mathrm{h}$. It should be noted that the above two saturation flow values are taken according to the recommended value of HCM 2010 [19]. The maximum deceleration is $-3.5 \mathrm{~m} / \mathrm{s}^{2}$; the minimum headway (front/real) is $1.0 \mathrm{~m}$. In this VISSIM simulation, right-turn vehicles that take exclusive lanes are not controlled by signals, and all pedestrians and bicycles are assumed to cross the road via elevated overpasses.

The model accuracy test follows the steps below.

Step 1. Change the cycle length of the roundabout. The maximum and minimum cycle lengths are set to be $160 \mathrm{~s}$ and $60 \mathrm{~s}$, that is, $\mathrm{T} \sim[60 \mathrm{~s}, 160 \mathrm{~s}]$, and the step size of the change is 10 s. The duration of each phase is $T / 4$, and the starting loss is $3 \mathrm{~s}$.

Step 2. With each cycle length, obtain the capacity of leftturn lanes by the following 3 approaches: VISSIM simulation, model proposed in this paper, and the model without the consideration of the internal space constraint.

Step 3. Compare the results of the above three approaches.

The model accuracy test result is shown in Table 2 . Using the results of VISSIM simulation as the benchmark and comparing the models with/without the consideration of internal space constraints, it is easy to observe that the model accuracy has been greatly improved when taking the internal space constraint into consideration. Results show that the relative error of the model proposed in this paper is basically no more than $10 \%$, of which the maximum is $13.94 \%$ and minimum is $1.54 \%$.

When calculating the capacity without the consideration of internal space constraint, the results have a large relative error compared with the VISSIM simulation, and the average error is $21.59 \%$. Compared with the model proposed in this paper, the capacity results without consideration of the 

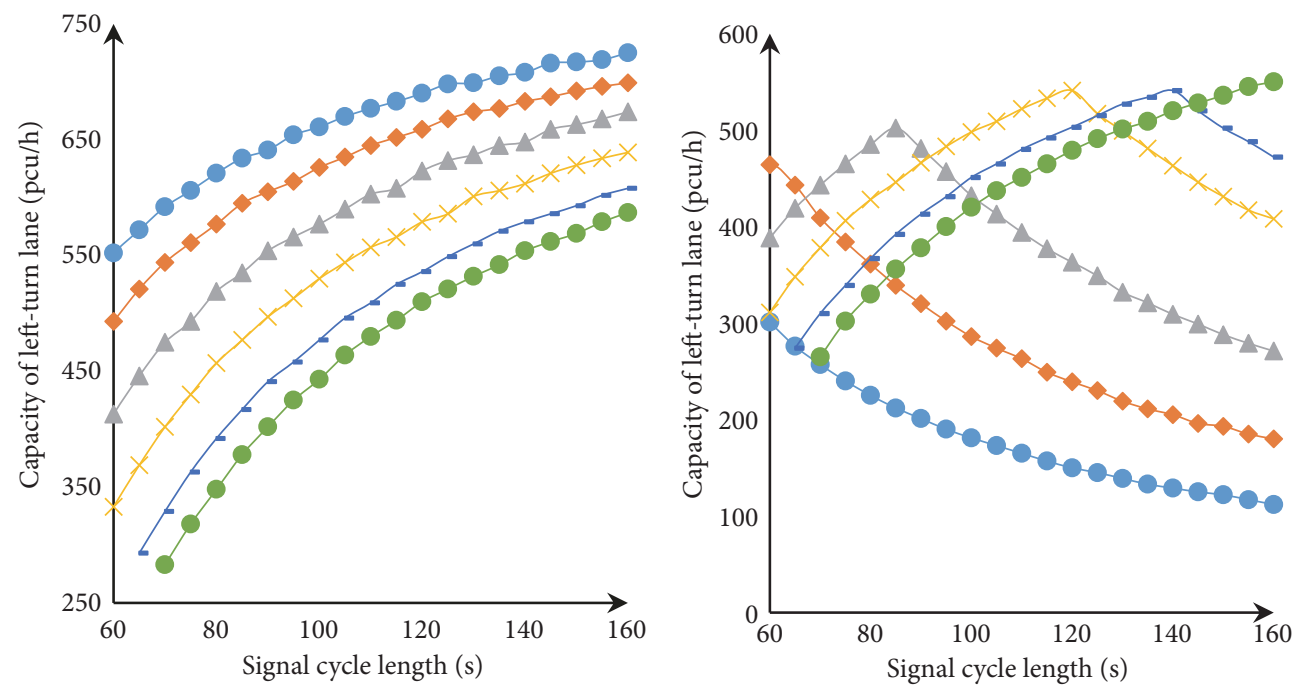

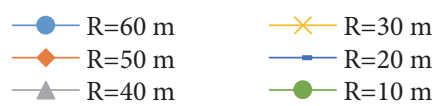

(a) Capacity of through lane

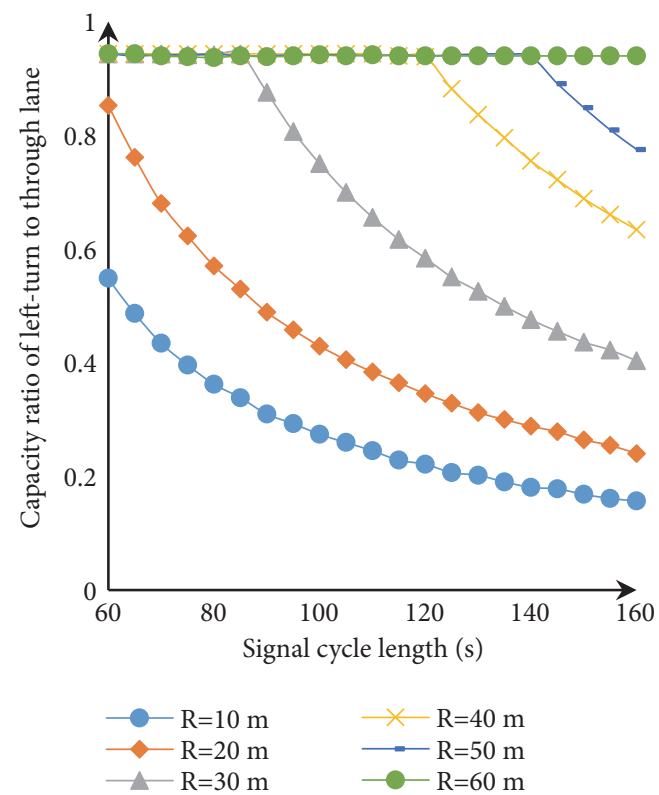

(c) Capacity ratio of left-turn lane to through lane

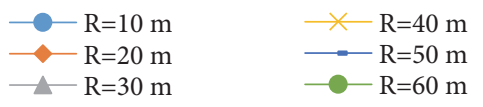

(b) Capacity of right-turn lane

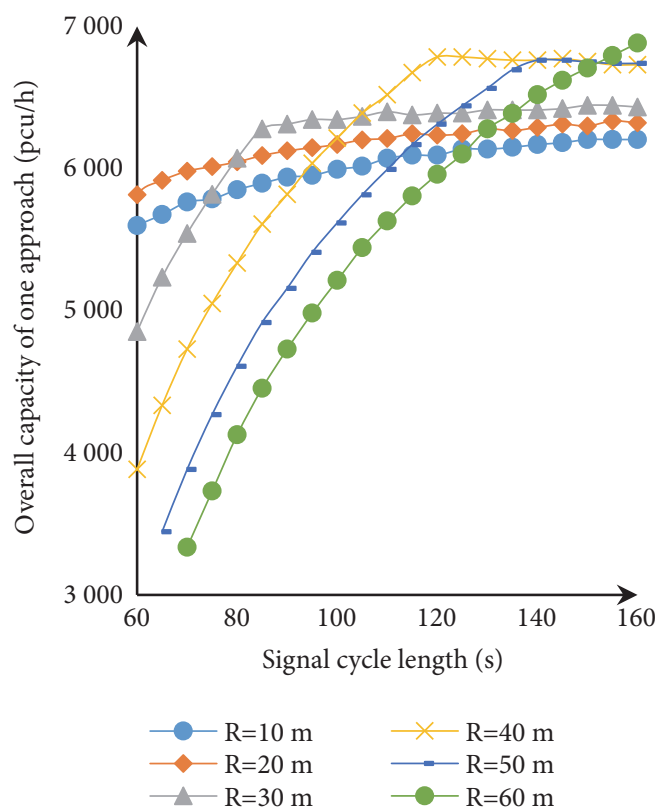

(d) Total capacity of approach

FIGURE 6: The sensitivity analysis results.

internal space constraint are relatively small. This is because if traffic signals are only equipped before the approach stop lines, the roundabout, at this time, is equivalent to a large-size signalized intersection, which requires a longer inter-green time to clean the intersection [21], and results in a longer green-lost time in one single cycle.

5.2. Sensitivity Analysis. Sensitivity analysis is also conducted to demonstrate the effect of different signal cycle lengths and central island radius on the capacity of a TSLSR. In this part, the signal cycle length varies from 60 s to 160 s, and the central island radius varies from $10 \mathrm{~m}$ to $60 \mathrm{~m}$. Figure 6 shows the results of sensitivity analysis.

As shown in Figure 6(a), the capacity of a through lane increases as the signal cycle length and the central island radius increase. This is because of the fact that longer signal cycle length means fewer signal switching interval resulting in less lost time. Moreover, when the central island radius is 
higher, the through traffic flow has a higher running speed in circulatory lanes; therefore, more vehicles can go through the roundabout.

Figure 6(b) illustrates the impacts of signal cycle length and central island diameters on the capacity of a left-turn lane. Similar to the through lane, the capacity of a left-turn lane increases with the central island radius getting larger. However, the cycle length has a relatively complicated effect, in which the capacity increases initially but then decreases after a threshold. When the signal cycle length is exactly equal to the threshold mentioned above, the left-turn traffic flow in one signal cycle length can just fulfill both the 1st and 2nd part of queuing areas. Moreover, if signal cycle length is less than the threshold, left-turn vehicles cannot fulfill the 1st and 2nd part of queuing areas, and the queuing areas cannot give full play to their capacity. If signal cycle length is longer than the threshold, spillback queues will occur and conflict with the entering vehicles in the following phase which then leads to a locking up of the roundabout. At the same time, this phenomenon also inspires us to infer that there is an optimal cycle length of TSLSR.

Figure 6(c) demonstrates the variation of the capacity ratio of left-turn to through lanes with respect to signal cycle length and central island diameters. This figure is obtained from the data shown in Figures 6(a) and 6(b). At last, one can also observe in Figure 6(d) that when the signal cycle length is lower, the overall capacity of one direction is sensitive to the cycle length. However, under high signal cycle length the overall capacity of one direction is almost unchanged.

\section{Optimal Cycle Length}

Cycle length is the most important and fundamental parameter in signal timing. Generally, for a signalized intersection, a longer signal cycle length means larger capacity and longer traffic delays. Therefore, according to the classic timing theory of signalized intersection, the optimal value of signal cycle length is mainly based on the overall minimum delay. For instance, in Webster method [22], the optimal cycle length is given by (7):

$$
T_{o p t}=\operatorname{argmin} \sum_{1}^{i} q_{i} \cdot d_{i}=\frac{1.5 L+5}{1-Y}
$$

As mentioned before, Figure 6(b) gives us inference that there is an optimal cycle length value, which is significantly affected by the interval space constraint. Therefore, the Webster method is not suitable for calculating the optimal signal cycle length of TSLSR, and an optimal cycle length value of a TSLSR can be obtained under the objective of capacity maximization as shown in Figure 7.

\section{Conclusion}

In this study, we proposed an engineering-oriented approach to calculate the capacity and optimal signal cycle length of the TSLSR, which is characterized by equipped signal lights on both the approaches and circulatory lanes and setting up of two stop lines for left-turn traffic flows.

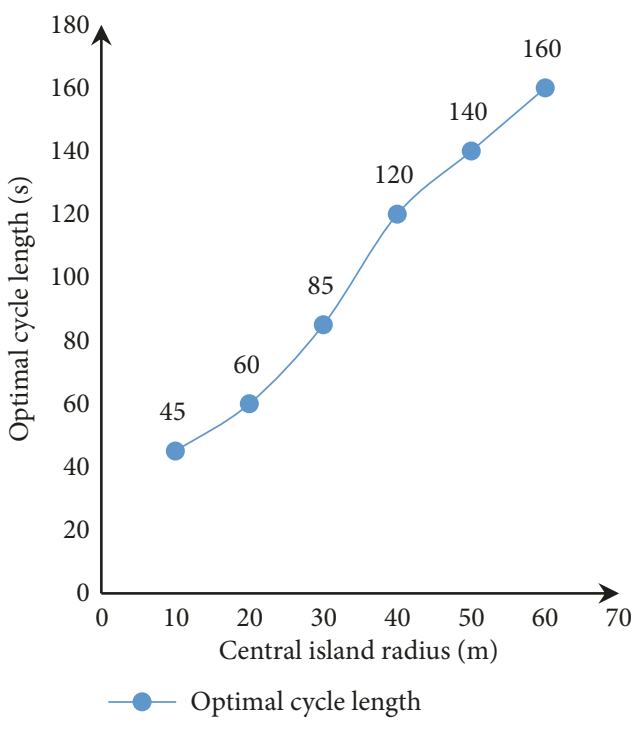

FIGURE 7: The optimal cycle length of TSLSR.

The operational logic of TSLSR is given in this paper which, for a better understanding, we demonstrate that it could be equivalent to a four-phase signalized intersection with left-turn protection phases. Furthermore, the left-turn lane capacity model could be simplified as the "short-lane model". Based on the above analyses, we gave the formula for calculating the capacity with the consideration of interval space constraint.

The accuracy test shows that the model proposed in this paper has a relatively high accuracy. The sensitivity analysis shows that there is a threshold point of left-turn capacity as the signal cycle length increases. Hence, different from the classic timing theory such as Webster method, an optimal cycle length of TSLSR can be obtained under the objective of capacity maximization.

In further research along the line, the impacts of pedestrians and bicycles on the capacity will be taken into consideration. Additionally, some fundamental parameters such as the saturation flow and green-lost time can be properly calibrated.

\section{Data Availability}

The data used to support the findings of this study are available from the corresponding author upon request.

\section{Conflicts of Interest}

The authors declare that there are no conflicts of interest regarding the publication of this paper.

\section{Acknowledgments}

This research is supported by the National Natural Science Foundation of China (61773293) and Chinese Government Scholarship (201806260148). The authors convey their sincere gratitude. 


\section{References}

[1] American Association of State Highway and Transportation Officials, Highway Safety Manual, AASHTO, Washington, DC, USA, 2010.

[2] People's Republic of China Ministry of Construction, Code for Transport Planning on Urban Road, People's Republic of China Ministry of Construction, Beijing, China, 1995.

[3] T. Tollazzi, R. Mauro, D. Zilioniene, I. I. Otković, and N. Stamatiadis, "Modern roundabouts: a challenge of the future," Journal of Advanced Transportation, vol. 2019, Article ID 3950891, 2 pages, 2019.

[4] K. Todd, "A history of roundabouts in britain," Transportation Quarterly, vol. 45, no. 1, pp. 143-155, 1991.

[5] N. Wu, "A universal procedure for capacity determination at unsignalized (priority-controlled) intersections," Transportation Research Part B: Methodological, vol. 35, no. 6, pp. 593-623, 2001.

[6] R. Guo, L. Liu, and W. Wang, "Review of roundabout capacity based on gap acceptance," Journal of Advanced Transportation, vol. 2019, Article ID 4971479, 11 pages, 2019.

[7] C. F. Daganzo, "Traffic delay at unsignalized intersections: clarification of some issues," Transportation Science, vol. 11, no. 2, pp. 180-189, 1977.

[8] D. Heidemann and H. Wegmann, "Queueing at unsignalized intersections," Transportation Research Part B: Methodological, vol. 31, no. 3, pp. 239-263, 1997.

[9] A. Pratelli, S. Casella, A. Farina et al., "Conventional and unconventional roundabouts: a review of geometric features and capacity models," International Journal of Transport Development and Integration, vol. 2, no. 3, pp. 225-239, 2018.

[10] J. Bie, H. K. Lo, and S. C. Wong, "Capacity evaluation of multilane traffic roundabout," Journal of Advanced Transportation, vol. 44, no. 4, pp. 245-255, 2010.

[11] P. Davies, B. Jamieson, and D. A. Reid, "Traffic signal control of roundabouts," Traffic Engineering And Control, vol. 21, no. 7, pp. 354-357, 1980.

[12] A. K. Patnaik, L. A. Agarwal, M. Panda, and P. K. Bhuyan, "Entry capacity modelling of signalized roundabouts under heterogeneous traffic conditions," Transportation Letters, pp. 113, 2018.

[13] P. Çalışkanelli, M. Özuysal, S. Tanyel et al., "Comparison of different capacity models for traffic circles," Transport, vol. 24, no. 4, pp. 257-264, 2009.

[14] F. F. Saccomanno, F. Cunto, G. Guido, and A. Vitale, "Comparing safety at signalized intersections and roundabouts using simulated rear-end conflicts," Transportation Research Record, vol. 2078, no. 1, pp. 90-95, 2008.

[15] X. Yang, L. Xiugang, Z. Guangwei et al., "The traffic control and management system for large roundabout," 2001.

[16] W. Ma, Y. Liu, L. Head, and X. Yang, "Integrated optimization of lane markings and timings for signalized roundabouts," Transportation Research Part C: Emerging Technologies, vol. 36, pp. 307-323, 2013.

[17] Z. Z. Tian and N. Wu, "Probabilistic model for signalized intersection capacity with a short right-turn lane," Journal of Transportation Engineering, vol. 132, no. 3, pp. 205-212, 2006.

[18] W. Ma, Y. Lu, K. An et al., "Probabilistic model for signalized intersection capacity with short lanes," Journal of Tongji University (Natural Science), vol. 40, no. 11, pp. 1641-1646, 2012.
[19] H. C. Manual, HCM2010, Transportation Research Board, National Research Council, Washington, DC, USA, 2010.

[20] E. Nyame-Baafi, C. A. Adams, and K. K. Osei, "Volume warrants for major and minor roads left-turning traffic lanes at unsignalized T-intersections: A case study using VISSIM modelling," Journal of Traffic and Transportation Engineering, vol. 5, no. 5, pp. 417-428, 2018 (English).

[21] Z. Jiang and T. Wang, "Intergreen time calculation method of signalized intersections based on safety reliability theory: a monte-carlo simulation approach," Journal of Advanced Transportation, vol. 2019, Article ID 1941405, 9 pages, 2019.

[22] Y. Wang, X. Yang, H. Liang, and Y. Liu, "A review of the selfadaptive traffic signal control system based on future traffic environment," Journal of Advanced Transportation, vol. 2018, Article ID 1096123, 12 pages, 2018. 


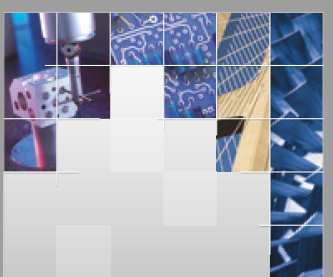

\section{Enfincering}
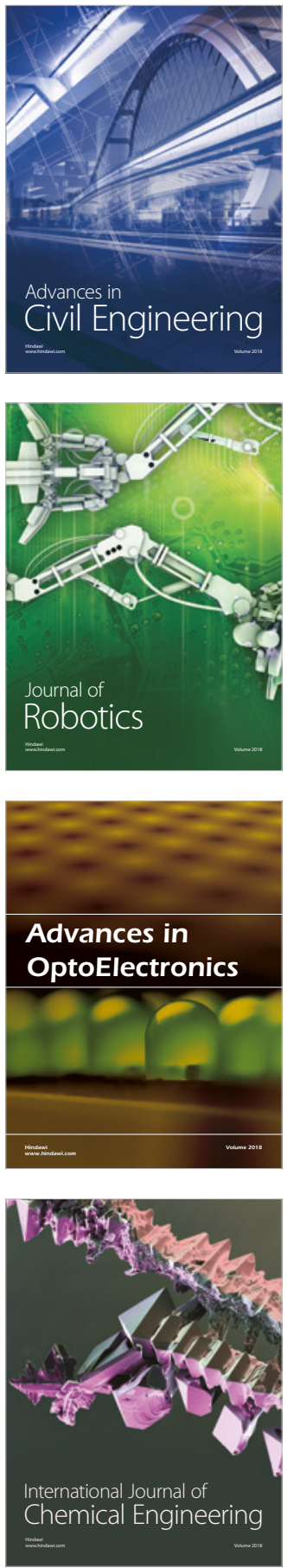

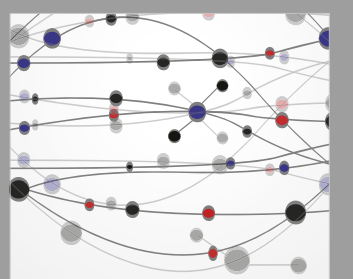

\section{Rotating \\ Machinery}

The Scientific World Journal

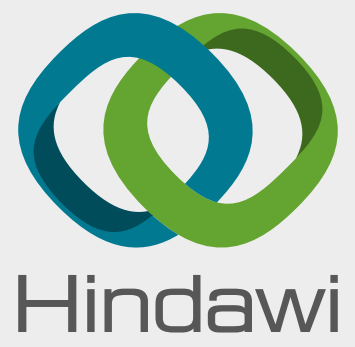

Submit your manuscripts at

www.hindawi.com
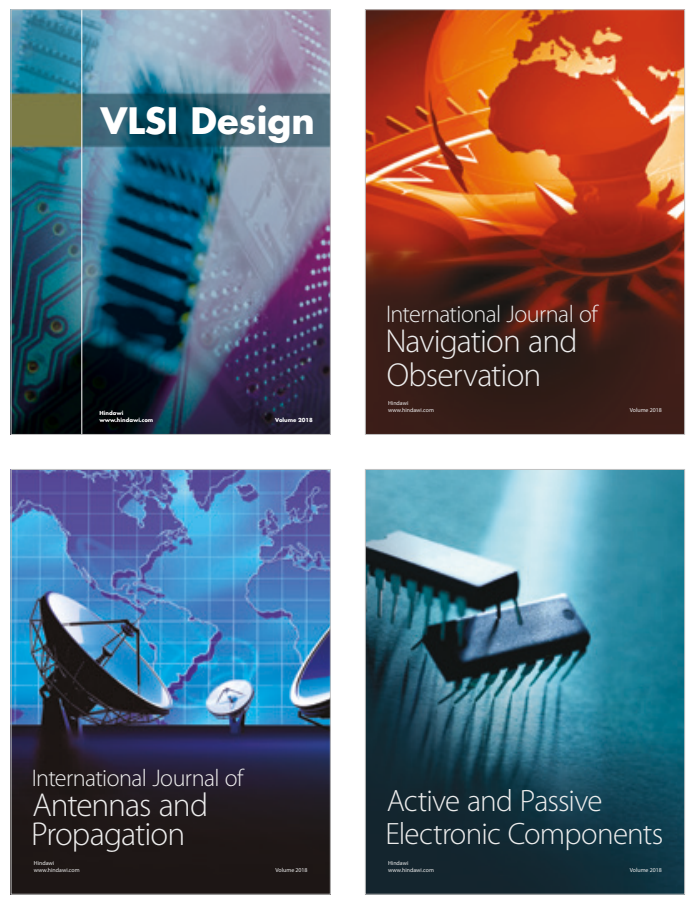
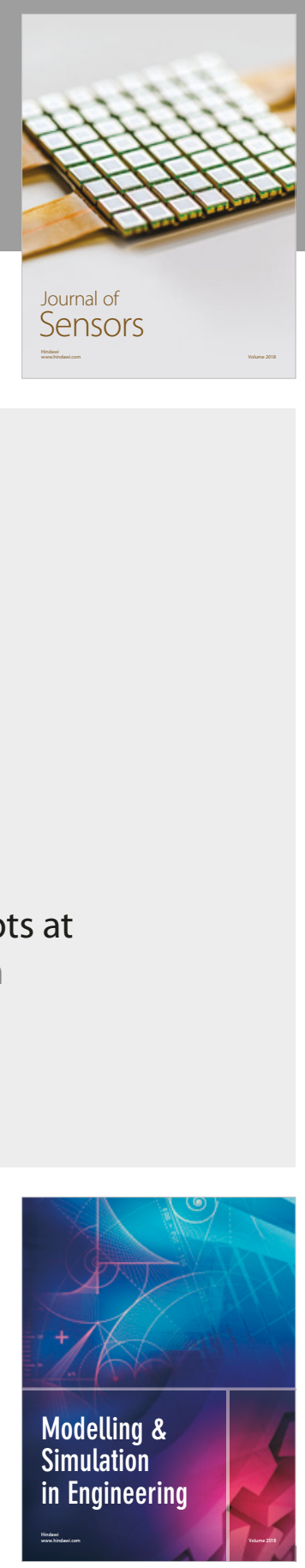

\section{Advances \\ Multimedia}
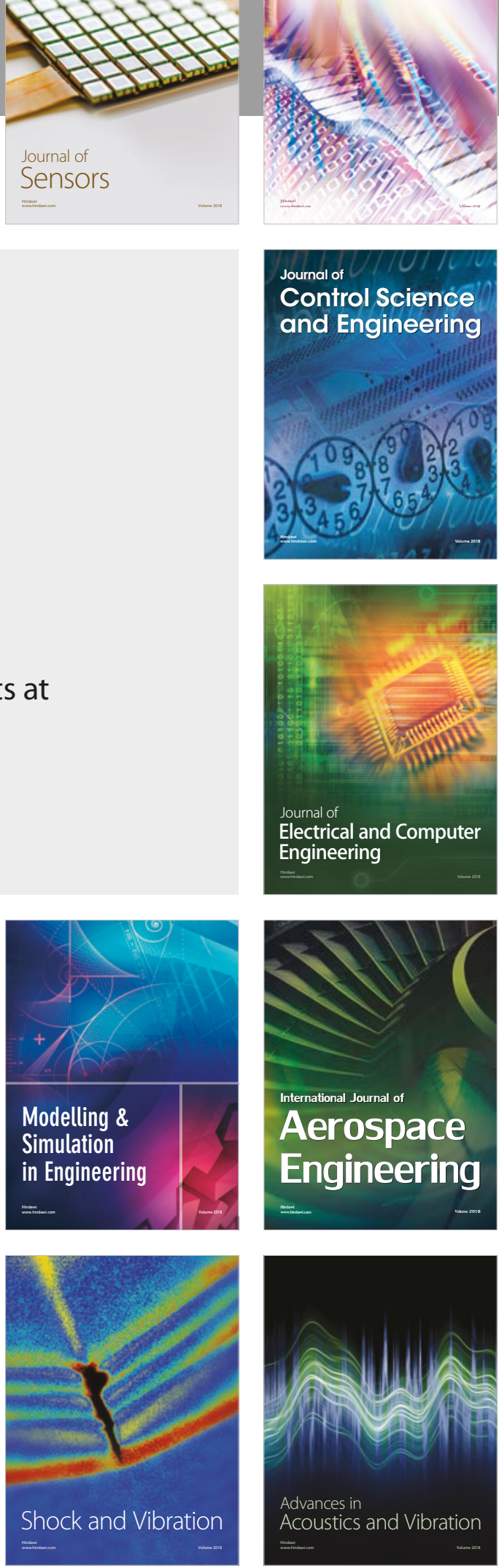